\title{
Caregiver teamwork improved psychotropic drug prescribing in nursing homes
}

Schmidt I, Claesson CB, Westerholm B, et al. The impact of regular multidisciplinary team interventions of psychotropic prescribing in Swedish nursing homes.J Am Geriatr Soc 1998 Jan;46:77-82.

\section{Question}

Does a multidisciplinary team intervention in addition to drug use guidelines affect psychotropic drug prescribing in nursing homes (NHs)?

\section{Design}

12 month randomised controlled trial.

\section{Setting}

$36 \mathrm{NHs}$ in Sweden.

\section{Patients}

1854 permanent NH residents (mean age 84 y, 70\% women) of whom $39 \%$ had a diagnosis of dementia, $4.3 \%$ had a psychotic disorder, and $6.4 \%$ had a diagnosis of depression. NHs were eligible if they were typical of the region, were under the jurisdiction of 1 of 18 randomly selected regional pharmacy directors, and the supervising physicians were not geriatric specialists.

\section{Intervention}

NHs were allocated to an experimental intervention group (15 $\mathrm{NHs}, 626$ residents) or a control group (18 NHs, 1228 residents). The experimental intervention involved 1 visit each month by a pharmacist who organised team meetings of the $\mathrm{NH}$ physician and nursing personnel to discuss drug use in individual residents. The aim was to minimise non-recommended drug use as defined by the guidelines of the Swedish Medical Product Agency (SMPA). Control group NHs received no special efforts to influence drug prescribing except the SMPA guidelines which were distributed to all physicians at the start of the intervention.

\section{Main outcome measures}

Quantitative outcomes were rates of psychotropic (antipsychotic, hypnotic, anxiolytic, and antidepressant) drug prescrib ing, polymedicine ( $\geqslant 2$ drug classes), and therapeutic duplication ( $\geqslant 2$ drugs in the same class); qualitative outcomes were recommended and non-recommended drug prescribing.

\begin{abstract}
Main results
Total rates of psychotropic drug prescribing, polymedicine, and therapeutic duplication did not change from baseline in experimental group NHs but in control group NHs therapeutic duplication increased by $17 \%(\mathrm{p}=0.04)$ and the mean number of drugs prescribed increased by 7\% $(\mathrm{p}=0.02)$. Prescription of antipsychotic drugs decreased by $19 \%(\mathrm{p}=0.007)$ in experimental group NHs compared with a non-significant decrease of $7 \%$ in control group NHs. In experimental group NHs, use of nonrecommended hypnotic drugs decreased by $37 \%(\mathrm{p}<0.001)$ and use of acceptable hypnotic drugs increased by $70 \%$ $(p<0.001)$; no change was seen in control group NHs. No change in prescription of non-recommended anxiolytic drugs was seen in either group but the prescription rate of acceptable anxiolytic drugs increased by $50 \%$ in experimental group $\mathrm{NHs}$ $(p=0.002)$. Statistically significant decreases in use of nonrecommended antidepressant drugs occurred in both experimental group NHs $(59 \%, \mathrm{p}<0.001)$ and control group $\mathrm{NHs}$ $(34 \%, \mathrm{p}=0.002)$ and use of recommended antidepressants increased by $584 \%$ and $315 \%$, respectively $(\mathrm{p}<0.001)$.
\end{abstract}

\section{Conclusion}

An intervention involving teamwork of physicians, pharmacists, and nurses decreased the prescribing of antipsychotic and nonrecommended hypnotic drugs in nursing homes.

Sources of funding: National Corporation of Swedish Pharmacies and Swedish Pharmaceutical Society.

For correspondence: Ms Ingrid Schmidt, Läkemedelsprojektet, The National Board of Health and Welfare, 10630 Stockholm, Sweden.Email Ingrid.Schmidt@sos.se.

\section{Commentary}

Psychotropic drugs should be used with care in the elderly because of adverse side effects including sedation, and undesirable interactions with medications for physical ilnesses. SMPA treatment guidelines for prescribing psychotropic drugs in the demented elderly were distributed in 1994 to all Swedish physicians, recommending minimal use of antipsychotics, certain benzodiazepines, and tricyclic antidepressants with anticholinergic properties. Schmidt et al examine the effect of the guidelines, thus extending various uncontrolled studies of psychotropic drug use in NHs in the US. ${ }^{1}$

The authors had to contend with different sizes of $\mathrm{NH}$ facilities and changes in residents. Firstly, despite randomisation, experimental NHs were smaller than control NHs (mean $51 v 80$ beds). Even where staff:resident ratios are equivalent, if all staff members care for all residents then home size necessarily affects the quality of social interactions. Such psychosocial factors contribute to behavioural and mental health problems. Secondly, there was a $10.2 \%$ reduction in experimental $\mathrm{NH}$ residents by 12 months compared with a $1.2 \%$ increase in control NH residents. Death rate or endpoint data by diagnostic category or functional status were not provided. Resident turnover may affect prescribing practice because those who died or moved home could also be those maintained on the older, non-recommended drugs; deaths can upset staff and residents, potentially increasing behavioural or mental health problems; and new residents may have been admitted because of behavioural or mental health problems. ${ }^{2}$
Education and support are necessary for NH staff members to implement nonmedication interventions for behavioural and mental health problems, ${ }^{3}$ and passive dissemination of guidelines tends not to change professional practice. ${ }^{4}$ Schmidt $e t$ $a l$ show that externally facilitated casereviews involving a pharmacist trained in communication skills, problem solving, and staff support are more successful than written guidelines alone.

Georgina Charlesworth, ClinPsyD, MPhil University of East Anglia Norwich, Norfolk, UK

1 Stoudemire A, Smith DA. Gen Hosp Psychiatry 1996;18:77-94.

2 Morriss RK, Rovner BW, German PS. Int J Geriatr Psychiatry 1996;11:243-9.

3 Rovner BW, Steele CD, Shmuely Y, et al. J Am Geriatr Soc 1996;44:7-13.

NHS Centre for Reviews and Dissemination. Effective Health Care 1994;8:1-12. 